\title{
Factores que contribuyen en la deserción de los estudiantes de la Escuela de Ciencias Administrativas en la UNAD - CEAD Simón Bolívar - Cartagena
}

\author{
William Del Toro Díaz ${ }^{1}$
}

\begin{abstract}
Resumen
La investigación realizada sobre la deserción académica en ECACEN, muestra los elementos más relevantes de esta problemática, basados principalmente en los siguientes factores: rendimiento académico, ingreso familiar, nivel académico, tipo de institución y la edad. La Escuela ECACEN del CEAD Simón Bolívar no es ajena a este fenómeno, las estadísticas de deserción, de los últimos dos años, arrojan los siguientes datos: para el periodo 2007-II al periodo 2008-I, la deserción fue del $37 \%$, para periodo 2008-II al 2009-I, el $29 \%$.
\end{abstract}

Para evitar la deserción de los estudiantes de la Escuela ECACEN en el CEAD Simón Bolívar - Cartagena, se han constituido muchas estrategias, entre ellas se puede destacar: las visitas a los sitios de reclusión, plan padrino, disponibilidad permanente de aula de sistemas en el CEAD, comunicación telefónica y electrónica, y eventos culturales. Todas estas estrategias se crearon sin tener el soporte de una investigación formal y exhaustiva de las causas que la generan. A pesar de todas las estrategias utilizadas, las cifras de deserción en ECACEN son muy elevadas; frente a esta situación la investigación identifica varios factores que contribuyen en la deserción de los estudiantes de la Escuela ECACEN de la UNAD.

Palabras clave: aprendientes, deserción, estadísticas, estrategias, investigación, problemática, rendimiento académico. 


\title{
Factors discouraging school attendance of students from administration science faculty UNAD-CEAD Simón Bolívar - Cartagena
}

\begin{abstract}
The study of school dropout in ECACEN, presents the most important elements of this problem mainly based in the light of the factors: academic performance, family income, academic level, type and age. The ECACEN CEAD Simón Bolívar School is not exempt from this phenomenon. The statistics of desertion from the last two years show the following data; from period 2007-II to period 2008-I the dropout was 37 $\%$, and for period 2008-II 2009-I drop was $29 \%$.

In order to avoid the desertion of students of the school in the ECACEN CEAD Simón Bolívar - Cartagena, several strategies have been implemented. Among these were included visits to the places of desertion, students support, continuous availability of classroom systems in the CEAD, telephone and electronic communication and cultural events. All these strategies were created without the support of a formal investigation and depth understanding of their root causes. Despite of all the strategies used the desertion in ECACEN is very high. In light with the situation this study identifies several factors contributing to the desertion of students of the ECACEN UNAD School.
\end{abstract}

Key words: learners, dropout, statistics, strategies, research, problem, academic performance.

Recibido: 4 de abril de 2013

Aceptado: 24 de junio de 2013

\section{Introducción}

La UNAD, en los últimos años, ha tenido una serie de transformaciones internas que obedecen al normal desarrollo y evolución de la institución y su proceso de modernización. Ha pasado de ser Institución Universitaria a ente autónomo y con la nueva administración se ha reorganizado y mejorado su estructura interna, tanto en el aspecto organizacional como académico. Igualmente, los avances en tecnología y el trabajo en red, han contribuido a constituir un organismo sólido, coherente y conectado, en la búsqueda permanente de la calidad institucional. 
La deserción no es ajena a la UNAD, que en concordancia con el contexto internacional y nacional, expresa en su Misión el "contribuir a la educación para todos..." y sus principios se fundamentan en "...la igualdad, la moralidad, la eficiencia, la economía, la celeridad, la imparcialidad, la participación democrática, la transparencia y la equidad social ". Aspectos generadores de cultura y espíritu emprendedor en el marco de una sociedad global y competitiva.

Según estudio realizado por el CEAD "Elementos culturales que influyen en la deserción estudiantil de la UNAD, CEAD Simón Bolívar Cartagena ". El fenómeno de la deserción afecta a la escuela ECACEN - CEAD Simón Bolívar, dado que todos sus programas son a distancia y en modalidad virtual, lo cual requiere que estudiante desarrolle su aprendizaje autónomo y significativo con mayor compromiso.

De acuerdo a lo anterior, en el presente documento el lector encontrará un informe de investigación donde se identificaron los factores que contribuyen a la deserción de los estudiantes en los programas de ECACEN, en el CEAD Simón Bolívar de Cartagena.

\section{Metodología}

La investigación es de tipo descriptiva, analítica y cuantitativa, porque buscaba identificar cuáles son los factores que contribuyen en la deserción de los estudiantes en la Escuela Ciencias Administrativa de la UNAD. Población. La población base de la Investigación son estudiantes del CEAD Simón Bolívar de diferentes estratos socioeconómicos que desertaron, y en su gran mayoría son becados por el distrito de Cartagena con características poblacionales vulnerables. La información personal y académica que se obtuvo de los estudiantes se encuentra en los archivos de la oficina de Registro y Control, como también información que tienen las consejeras académicas del CEAD.

Para obtener el tamaño de la muestra se tuvo presente una población total de 101 estudiantes que desertaron en el segundo periodo de 2008 y que se mantienen en estado de deserción académica, para el primer periodo del 2009 en ECACEN - Simón Bolívar; los estudiantes son de diferentes estratos socioeconómicos que ingresan a diferentes programas de la Escuela. La muestra se calculó a través de la ecuación de Harvard, utilizando la proporción, en donde se asume el valor máximo posible que podría tomar la varianza, que es cuando el valor de $p=0,5$ ya que su formula es $p$ (probabilidad de éxito) y $q$ (probabilidad de fracaso), y sigma cuadrado es igual a p por q. Cuando p y q son iguales, este resultado corresponde al máximo valor posible que podría tomar a la varianza. 


\section{William Del Toro Díaz}

Factores que contribuyen en la deserción de los estudiantes de la Escuela de Ciencias Administrativas en la UNAD - CEAD Simón Bolívar - Cartagena. Artículo original producto de la investigación.

E: error permitido: $5 \%$

Z: 1,96

$\mathrm{P}=\mathrm{q}=0.5$

Nivel de confianza $95 \%$

N: Población

$n$ : Muestras aplicar

$n=\frac{p^{*} q^{*} N^{*} 1,96}{E^{2 *}(N-1)+\mathrm{z}^{*} p^{*} q}$

Desarrollando la ecuación el resultado de las muestras a aplicar es el siguiente:

$$
\begin{aligned}
& n=\frac{0.5 * 0.5 * 101 * 1.96}{0.05 * 0.05 *(101-1)+1.96 * 0.5 * 0.5} \\
& n=\frac{49.49}{0.74}=66.878 \\
& n=67
\end{aligned}
$$

El número de la muestra a la que se aplicó un instrumento de obtención de información en nuestro proyecto de investigación, corresponde a 67 estudiantes, así:
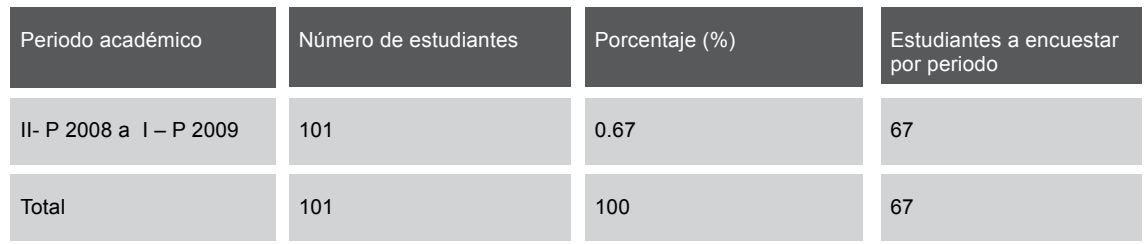

La selección de los estudiantes a encuestar está comprendida entre el II periodo de 2008 y el I periodo de 2009, se hizo a través de un muestreo aleatorio simple, teniendo como referencia los listados de los estudiantes que desertaron, información de su ubicación actual se encuestaran en los archivos de la oficina de registro y control y la información de los mismos que tiene la Consejeria del CEAD, otorgando a todos los estudiantes la misma probabilidad de ser elegidos de manera al azar.

En cuanto a la técnica, la información recolectada en la investigación se realizó a partir de fuentes primarias por medio de la aplicación de encuestas tipo cuestionario, con preguntas semiestructuradas y que fueron aplicadas, 


\section{Revista de}

por medio del trabajo colaborativo, por dos estudiantes. Los estudiantes seleccionados, a quienes se les aplicó el cuestionario fueron contactados personalmente y otros vía celular, informándoles que diligenciaran el instrumento para recolección de información.

\section{Resultados y discusión}

Periodo: de acuerdo con los resultados se puede observar que del total de estudiantes encuestados, el $25 \%$ pertenece a la Escuela al periodo 2, el $21 \%$, al periodo 3 y para los periodos 4,5 y 6 el porcentaje de participación es del $18 \%$ para cada uno.

\begin{tabular}{|l|l|l|}
\hline $\begin{array}{l}\text { Periodo en que se } \\
\text { encuentra matriculado }\end{array}$ & Total & Porcentaje (\%) \\
\hline 2do período & 17 & $25 \%$ \\
\hline 3er período & 14 & $21 \%$ \\
\hline 4to período & 12 & $18 \%$ \\
\hline 5to período & 12 & $18 \%$ \\
\hline 6to período & 12 & $18 \%$ \\
\hline & 67 & $100 \%$ \\
\hline
\end{tabular}

Edad: los resultados muestran que el 52\% de los estudiantes tiene edades entre 20 - 30 años, el 22\% tiene entre 30 - 40 años, el 18\% tiene mas de 40 años y solamente el $7 \%$, esta comprendido en edades de 15 - 20 años.

Estado civil: de acuerdo con los resultados se puede observar que el $46 \%$ de los encuestados son solteros, el 25\% son casados, el 19\% viven en unión libre, el $6 \%$ son divorciados y el $3 \%$ separados.

\section{Ubicación tecnológica del aprendiente}

\section{¿Tiene computador propio?}

El $61 \%$ de los encuestados manifiestan que tienen un computador propio, mientras que el 39\% restante no cuentan con un computador propio en su casa. 


\section{William Del Toro Díaz}

Factores que contribuyen en la deserción de los estudiantes de la Escuela de Ciencias Administrativas

en la UNAD - CEAD Simón Bolívar - Cartagena. Artículo original producto de la investigación.

\section{¿Desde dónde accede al internet?}

Los resultados muestran que el $48 \%$ de los encuestados manifiesta que tiene acceso a Internet desde su casa, el 25\% usa un café internet, el 12\% utiliza el internet de la oficina, el 7\% no tiene acceso fácilmente, el $6 \%$ usa las instalaciones de la UNAD, y solamente el $1 \%$ utiliza el servicio de un familiar

\section{Nivel educativo del aprendiente}

\section{¿Hace cuánto que terminó el bachillerato?}

El 36\% de los encuestados manifiesta que tienen entre 1 y 2 años de haber terminado bachillerato, el 34\% tiene menos de un año de haber terminado, el $16 \%$ manifiesta que tienen entre 2 y 3 años y el 13\% más de tres años.

\section{¿Está cursando estudios en otra institución educativa?}

El 93\% de los estudiantes manifiesta que no están estudiando en otra Institución de Educación Superior y el 7\%, afirma que si.

\section{Nivel familiar del aprendiente}

\section{¿Cuántas personas tiene a su cargo?}

El 34\% de los estudiantes manifiesta que no tienen bajo su responsabilidad ninguna persona, el $31 \%$ entre una y dos personas a cargo, el $19 \%$ entre 3 - 4 persona a su cargo y el 15\% manifiesta tener más de cuatro personas a cargo.

\section{¿Cuántos hijos tiene?}

El $52 \%$ de los encuestados manifiesta que tiene entre 1 y dos hijos, el $31 \%$ no tiene ninguno, el $10 \%$ tiene entre 3 y cuatro hijos y solo $6 \%$ tiene más de cuatro hijos.

\section{Nivel económico}

\section{¿Cuenta con el apoyo económico de su familia?}

El $76 \%$ de los encuestados manifiesta que no tiene apoyo económico de la familia, y el $24 \%$ afirma que si.

\section{¿Usted trabaja?}

Aproximadamente el $72 \%$ de los encuestados manifiesta que si están actualmente trabajando y un $28 \%$, no tiene trabajo.

\section{Ingresos de la familia}

De acuerdo con los resultados observados, el 54\% de los encuestados manifiesta que los ingresos familiares son entre $\$ 300.000-\$ 800.000$, el 
$28 \%$ dice que los ingresos son menores de $\$ 300.000$, el $15 \%$ entre 800.000 y 1.200 .000 y solo el 3\% gana más de 1.200 .000 .

\section{Aspecto académico y consideraciones sobre la metodología educativa}

\section{¿Para usted la educación a distancia se puede definir como?}

Los resultados especifican que el $45 \%$ de los encuestados cree que la educación a distancia es una metodología que hace uso de las Tic para su estudio, un $40 \%$ piensa que es un sistema de estudio que requiere del estudiante su aprendizaje autónomo e independiente y el 13\% que el sistema de estudio es igual a la presencial.

\section{¿Qué tipo de mediación prefiere usted?}

Los resultados especifican que el $37 \%$ de los encuestados prefiere la mediación a distancia, el $30 \%$ Semi- presencial, el $22 \%$ presencial y al $10 \%$ no le interesa el tipo de mediación.

¿La carrera que escogió llena las expectativas que usted se planteó para el inicio de la misma?

E1 78\% de los encuestados manifiesta que la carrera que estaba estudiando llenó sus expectativas, mientras que el $22 \%$ considera que no llenó sus expectativas.

¿Qué dificultades de 1 a 3 siendo 1 la primera y 3 la última experimentó usted y fueron motivo para su retiro?

El 64\% de los encuestados manifestó que la mayor dificultad que tuvieron fue que no pudieron administrar bien el tiempo; como segunda opción se encuentra dificultades con el manejo del computador, representado en un $34 \%$.

¿Qué dificultades de 1 a 3 siendo 1 la primera y 3 la última experimento Usted y fueron motivo para su retiro?

Los encuestados manifestaron que la inflexibilidad laboral, representada en un $66 \%$ fue otra de las razones que los llevaron a retirarse de los estudios; consideran también con un $31 \%$ la estrechez económica.

\section{¿Usted considera que la causa de su retiro fue la desmotivación?}

Para la pregunta (17) se puede observar que los encuestados manifiestan que si tuvo que ver la desmotivación para retirarse de la universidad, representado este valor en un $79 \%$.

¿La metodología ofrecida por la universidad influyó en las razones para su retiro?

El 55\% anotó que la metodología no fue una razón para retirarse de la universidad, pero un $45 \%$ dice que sí. 
Factores que contribuyen en la deserción de los estudiantes de la Escuela de Ciencias Administrativas en la UNAD - CEAD Simón Bolívar - Cartagena. Artículo original producto de la investigación.

$¿$ Cree usted que su entorno o ambiente en el que se desenvuelve influyo en el retiro de la universidad?

Se puede observar, de acuerdo con los resultados que arrojó la encuesta, que solo el $72 \%$ considera que el entorno en donde se desenvuelve, tuvo que ver con el retiro de la universidad.

¿Cuáles de las siguientes características culturales han influido en el retiro temporal de sus estudios?

El 63\% de los encuestados manifestó que otro motivo que los llevó a tomar la decisión de no continuar con sus estudios fue el no saber trabajar en equipo, el $22 \%$ por haberse dedicado al folclorismo, el emprendimiento y la tranquilidad con un $5 \%$ cada uno.

¿Considera usted que el acompañamiento tutorías en el campus virtual fue apropiado a sus necesidades?

Los encuestados consideran, con un 70\%, que el acompañamiento en el campus virtual fue apropiado y un $30 \%$ no está satisfecho con el mismo.

¿La herramienta utilizada como mediación virtual (campus virtual) favoreció el proceso de aprendizaje?

Los encuestados consideran que la herramienta utilizada como mediación virtual (campus virtual) si favoreció su proceso de aprendizaje, representado en el $79 \%$, mientras que al $21 \%$ no le pareció apropiada.

¿La interacción entre los actores (tutor- director-estudiante) del curso se presento de manera oportuna en cuanto a tiempo y a solución de inquietudes? Los encuestados están de acuerdo con un 67\% que la interacción con tutordirector-estudiante se presentó de manera oportuna favoreciendo de esta manera su proceso de enseñanza-aprendizaje y un 33\%, manifestó que no se presentó.

¿Si le dieran la oportunidad de ingresar nuevamente a la Universidad Nacional Abierta y a Distancia UNAD lo haría? ¿Por qué?

De acuerdo con los resultados se puede observar que el $82 \%$ de los encuestados estarían dispuestos a continuar con sus estudios si la Universidad les diera la oportunidad de regresar nuevamente y el $18 \%$ dice que no.

¿Considera que los materiales didácticos (Módulos, guías de trabajo) utilizados por la UNAD, son: completos, pertinentes o desactualizados?

De acuerdo con los resultados se puede observar que los encuestados manifiestan con un 91\% que los materiales didácticos (módulos, guías de trabajo) se encuentran completos, el $88 \%$ dice que son pertinentes, mientras que el $88 \%$ manifiesta que no se encuentran desactualizados. 


\section{¿Qué sugiere usted para mejorar en la educación a distancia?}

De acuerdo con los resultados al $28 \%$ de los estudiantes les gustaría contar con mayor interacción con los compañeros, el 22\% prefiere más tutorías presencia, el $22 \%$ que exista mas contacto con los tutores virtuales, un $15 \%$ solicita mayor capacitación en campus y el $12 \%$ manifiesta más acompañamiento de las consejeras.

\section{Conclusiones}

Las conclusiones que arroja la investigación fueron las siguientes: la mayoría de los estudiantes son adultos, solteros, pero también trabajan, lo que indica que no cuentan con el tiempo requerido para dedicarle al desarrollo de los cursos en el campus, donde tienen matriculados de 12 a 14 créditos académicos. Teniendo en cuenta el modelo pedagógico de la UNAD, basado en el aprendizaje autónomo, por lo general un estudiante necesitaría más tiempo del que un estudiante en el sistema tradicional usa para apropiarse de los conocimientos de los cursos. Esta podría ser una variable importante que contribuiría en la deserción de los estudiantes.

Los estudiantes No tienen dificultades para acceder al campus, ya que cuentan en sus casas con computadores y la conectividad requerida para la realización de las distintas actividades propuestas en cada unos de los cursos matriculados para el periodo.

Dentro de los factores que influyen en la deserción estudiantil podemos destacar el factor económico, la mala administración del tiempo por parte del estudiante así como la falta de apoyo permanente para el desarrollo de su proceso de aprendizaje por parte de sus líderes laborales; este factor contribuye a la deserción de sus actividades académicas, debido a que no quieren tener inconvenientes en sus responsabilidades laborales; también manifiestan que les gustaría tener mas contacto con los tutores presenciales y virtuales, lo que originaria una menor probabilidad de abandono de sus estudios.

Después de revisar y analizar los resultados obtenidos, es importante iniciar el proceso de desprenderse definitivamente del modelo tradicional (la educación presencial) y presentar el modelo de educación a distancia, como la educación del presente y del futuro, donde el estudiante cada día entienda más su real significado.

Que los estudiantes, tutores, consejeros y administrativos, jueguen su rol verdadero en el modelo de educación abierta y a distancia y generen las 


\section{William Del Toro Díaz}

Factores que contribuyen en la deserción de los estudiantes de la Escuela de Ciencias Administrativas

en la UNAD - CEAD Simón Bolívar - Cartagena. Artículo original producto de la investigación.

estrategias significativas para que la educación abierta y a distancia, se convierta en la alternativa, de todas aquellas personas que quieren mejorar su nivel educativo y por ende su nivel de vida, desde cualquier rincón del mundo.

Las estrategias utilizadas por el CEAD Simón Bolívar, como el plan padrino (A cada actor académico se le asigna un numero de estudiante, a los cuales tienen que hacerle un seguimiento y lograr que no abandonen sus estudios), son validas. Se recomienda una mayor socialización y medir sus resultados e impacto en la comunidad académica de la ciudad.

Finalmente, el Dr. José Manuel Restrepo Abondano en su artículo La calidad de la educación superior y la deserción estudiantil: frenos a la competitividad de Colombia, afirma que "La articulación entre el sector productivo colombiano y las instituciones de educación superior todavía no logra impactar la competitividad del país, mientras la deserción estudiantil abre aún más la brecha social entre jóvenes profesionales y jóvenes desempleados ".

\section{Referencias bibliográficas}

Clavijo, D. (2000). Educación superior en la década 1990-1999: Resumen Estadístico. Ministerio de Educacion Nacional de la Republica de Colombia. Consultado: 24 de Octubre de 2009, desde http://www.mineducacion.gov.co/1621/articles-85665_ archivo_pdfA2.pdf

Guzmán, C. (2006), Caracterización de la deserción estudiantil en la universidad Nacional de Colombia. Sistema de Prevención y Análisis de la Deserción en Las Instituciones de Educación Superior (SPADIES). Extraído 24 de Octubre de 2009, desde

Himmel, E (2002). Modelos de Análisis de la deserción estudiantil en la Educación Superior. Extraído 24 de Octubre de 2009, desde www.cse.cl/public/secciones/.../ doc/35/cse_articulo141.pdf

http://spadies.uniandes.edu.co/spadies2/recursos/CEDE_ProyectoDesercion.pdf

Ministerio de Educación Nacional. (2002). Estudio de la deserción estudiantil en la educación superior en Colombia. Universidad pedagógica y tecnológica de Colombia. Extraído 24 de Octubre de 2009, desde http://www.uptc.edu.co/export/descargas autoevaluacion/d8.pdf 


\section{Revista de}

Ministerio de Educación Nacional. (2006) Diagnóstico de la deserción estudiantil en Colombia. Extraído 24 de Octubre de 2009, desde http://menweb.mineducacion.gov. co/educacion_superior/numero_07/001.htm

Ramos, N. (2003). Deserción universitaria. Universidad Andina. Extraído 22 de diciembre de 2008, desde http://www.monografias.com/trabajos44/desercion-universitaria/ desercion-universitaria.shtml

Rico, D. (2006). Caracterización de la deserción estudiantil en la universidad Nacional de Colombia sede Medellín. Universidad Nacional de Medellín. Extraído 18 de diciembre de 2008, desde http://www.unalmed.edu.co/acreditacion/Factor\%202/Caracteristica7/ Estudio_de_la_Desercion_Estudiantil_Unalmed.pdf La calidad de la educación superior y la deserción estudiantil: frenos a la competitividad de Colombia. 


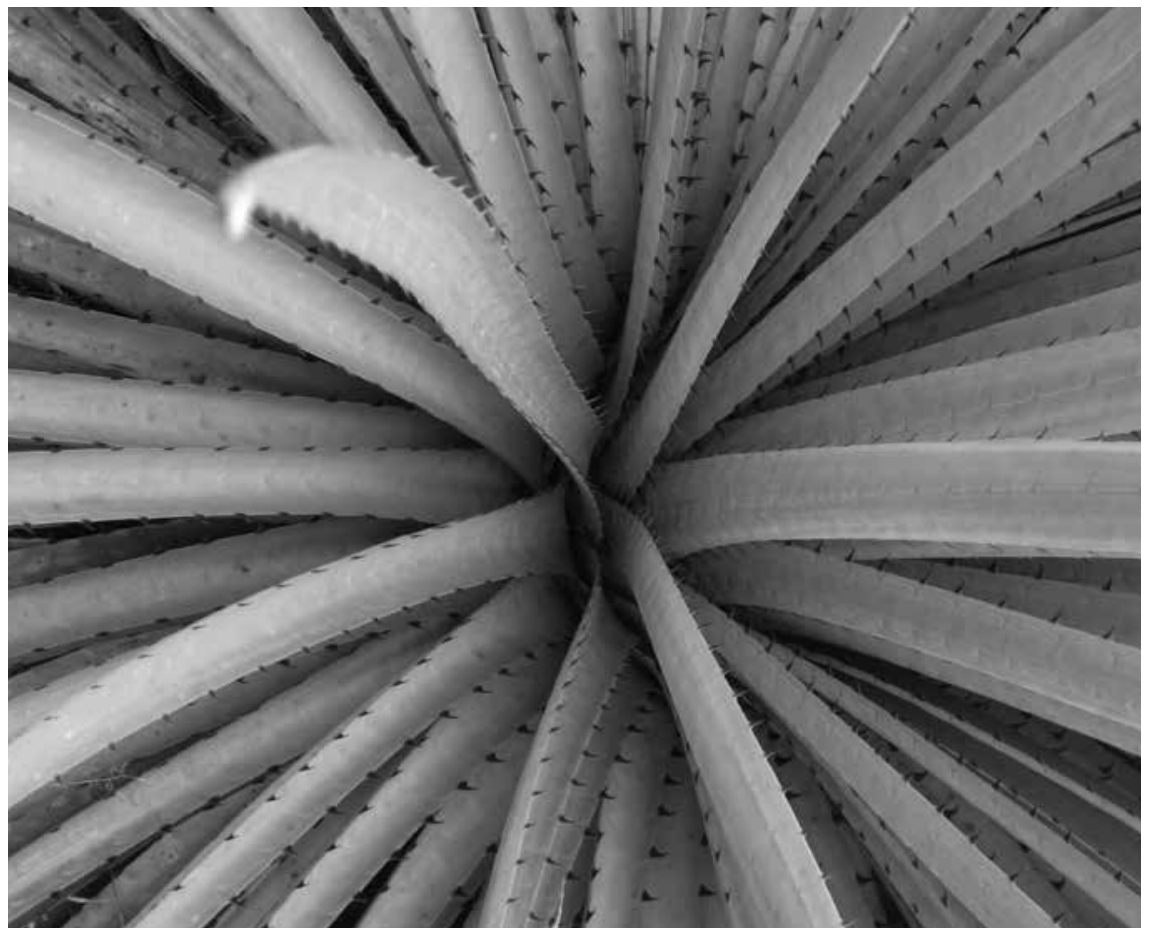

Fotografia: José Enrique Castillo 